\title{
Oscillations in pedunculopontine nucleus in Parkinson's disease and its relationship with deep brain stimulation
}

\author{
Min Li and Wangming Zhang* \\ The National Key Clinic Specialty, Guangdong Provincial Key Laboratory on Brain Function Repair and Regeneration, The \\ Engineering Technology Research Center of Education Ministry of China, Department of Neurosurgery, Zhujiang Hospital, \\ Southern Medical University, Guangzhou, China
}

\section{OPEN ACCESS}

Edited by:

Nicholas C. Spitzer, University of California, San Diego,

USA

Reviewed by: Michael M. Halassa, New York University, USA Antón Barreiro-Iglesias, University of Santiago de Compostela, Spain

*Correspondence: Wangming Zhang, The National Key Clinic Specialty, Guangdong Provincial Key Laboratory on Brain Function Repair and Regeneration, The Engineering

Technology Research Center of Education Ministry of China,

Department of Neurosurgery, Zhujiang Hospital, Southern Medical University, No. 253 Industrial Avenue, Haizhu District, Guangzhou 510282,

China wzhang@vip.126.com

Received: 27 June 2015 Accepted: 21 August 2015 Published: 02 September 2015

Citation:

Li M and Zhang W (2015) Oscillations in pedunculopontine nucleus in

Parkinson's disease and its relationship with deep brain stimulation.

Front. Neural Circuits 9:47. doi: 10.3389/fncir.2015.00047
The recent development of deep brain stimulation (DBS) of the pedunculopontine nucleus (PPN) for the treatment of parkinsonian patients, particularly those in advanced stages with axial symptoms, has ignited interest into the study of this brain nucleus. In contrast to the extensively studied alterations of neural activity that occur in the basal ganglia in Parkinson's disease (PD), our understanding of the activity of the PPN remains insufficient. In recent years, however, a series of studies recording oscillatory activity in the PPN of parkinsonian patients have made important findings. Here, we briefly review recent studies that explore the different kinds of oscillations observed in the PPN of parkinsonian patients, and how they underlie the pathophysiology of PD and the efficacy of PPN-DBS in these disorders.

Keywords: pedunculopontine nucleus, oscillations, Parkinson's disease, local field potentials, deep brain stimulation

\section{Introduction}

The use of single unit or action potential recordings to examine increases or decreases in neural firing does not fully capture the dysfunction of the basal ganglia in Parkinson's disease (PD). In recent years, the use of local field potential (LFP) recordings has made a resurgence in the investigation of the pathophysiology of Parkinsonian and other movement disorders. LFP, similar to EEG, is a kind of oscillatory activities, which is classified by the oscillatory frequency. Generally, LFP is divided into five bands: delta band $(1-3 \mathrm{~Hz})$, theta band $(4-7 \mathrm{~Hz})$, alpha band $(8-13 \mathrm{~Hz})$, beta band $(14-30 \mathrm{~Hz})$, and gamma band $(>30 \mathrm{~Hz})$. The boundaries of these bands may be a little different in studies. Patients' LFP is recorded through the electrode used for deep brain stimulation (DBS) after electrode implantation and before connection to the stimulator. In animals, special metal microelectrodes are planted into brain to record LFP. Several bands of oscillatory activities have been detected in the cortico-basal ganglia circuits, including in the subthalamic nucleus (STN) and the internal segment of the globus pallidus (Brown, 2003; Boraud et al., 2005; Gatev et al., 2006; Uhlhaas and Singer, 2006; Eusebio and Brown, 2007). Among these, aberrant beta band oscillations have been deemed to be one of the most important findings in the brain of PD patients (Levy et al., 2002; Fogelson et al., 2006; Weinberger et al., 2006; Chen et al., 2007; Hammond et al., 2007). These beta band abnormalities are believed to have anti-kinetic effects, and are likely to be responsible for some PD symptoms (Brown et al., 2001; Fogelson et al., 2006). 
In advanced stages of PD, axial symptoms such as severe gait and postural impairments that are not ameliorated by levodopa and STN stimulation present difficult obstacles for many patients. To relieve these symptoms, targeting the pedunculopontine nucleus (PPN) with DBS holds some promise (Moro et al., 2010; Thevathasan et al., 2011; Wilcox et al., 2011; Morita et al., 2014). Although the PPN generally is not considered part of the cortico-basal ganglia loop, there are extensive reciprocal connections between the PPN and the basal ganglia. Therefore, the question arises of whether the PPN may exhibit the same oscillatory activity as the basal ganglia in PD. Also, what is the relationship between PPN oscillations and the pathophysiology of PD? How are PPN oscillations altered with DBS treatment? These are the questions that will be discussed in this review.

\section{Overview of Oscillations in the PPN}

Activity in the alpha frequency has been the most noticeable oscillatory activity recorded in the PPN, first recorded in parkinsonian patients by Androulidakis et al. (2008a,b). Although alpha band oscillations have drawn less attention in studies of the basal ganglia, these activities likely play a pivotal role in the function of the PPN. For example, alpha band power increases significantly following treatment with levodopa (Androulidakis et al., 2008b; Fraix et al., 2013) and correlates with gait performance in PD patients (Thevathasan et al., 2012; Tattersall et al., 2014). These studies suggest that activity in the alpha band plays a physiological function in the PPN, and is pathologically attenuated in $\mathrm{PD}$.

Unlike findings in the cortical-basal ganglia loop, there currently exists no consensus as to the importance of beta band PPN activity in PD. A number of studies have shown that dopaminergic medication has a suppressive effect upon beta activity in the PPN, implying that this activity may contribute to akinesia in PD (Thevathasan et al., 2012; Fraix et al., 2013). Other studies have found quite the contrary, however. For example, beta oscillations in the PPN were shown to decrease in the absence of medication, but increase in the presence of medication, in patients making voluntary movements. In this study, beta coherence between the midline prefrontal region and the PPN was only found in the medicated state (Tsang et al., 2010). Thus, beta rhythms may have a different functional significance in the PPN compared with the basal ganglia, in that these oscillations could be prokinetic.

Topological differences appear to exist between the alpha and beta oscillations of the caudal vs. the rostral PPN. In parkinsonian patients, alpha oscillations represented the main frequency in the caudal subregion of the PPN, while beta oscillations predominate in the rostral subregion (Figure 1A; Weinberger et al., 2008; Thevathasan et al., 2012; Tattersall et al., 2014).

Theta and gamma band oscillations have also been recorded in the PPN (Shimamoto et al., 2010; Tsang et al., 2010; Fraix et al., 2013; Valencia et al., 2014; Lau et al., 2015). In the basal ganglia, theta activity has been suggested to underlie parkinsonian tremor, which appears and disappears in synchrony with muscle tremor (Stein and Bar-Gad, 2013). In the PPN, theta oscillations may be involved in the feedback of sensory information between the PPN and the sensorimotor cortices (will be discussed below; Tsang et al., 2010). Gamma oscillations were first reported in the PPN of parkinsonian patients by Fraix et al., who proposed that their functional impact might be similar to beta oscillations (Fraix et al., 2013). This certainly requires further investigation. Table $\mathbf{1}$ shows different studies looking at oscillatory activity in the PPN. The major oscillations in the PPN and their functional characteristics are summarized in Figure 1D and will be discussed below.

TABLE 1 | Different studies looking at oscillatory activity in the PPN.

\begin{tabular}{lll}
\hline Study & Subjects & Frequency band \\
\hline $\begin{array}{l}\text { Androulidakis et al. (2008a) } \\
\text { Androulidakis et al. (2008b) }\end{array}$ & $\begin{array}{l}\text { 1 patient } \\
6 \text { patients }\end{array}$ & $\begin{array}{l}\text { Alpha }(7-11 \mathrm{~Hz}) \\
\text { Alpha }(7-11 \mathrm{~Hz})\end{array}$ \\
$\begin{array}{l}\text { Weinberger et al. (2008) } \\
\text { Tsang et al. (2010) }\end{array}$ & $\begin{array}{l}\text { Beta }(15-30 \mathrm{~Hz}) \\
\text { Theta }(6-10 \mathrm{~Hz})\end{array}$ \\
Thevathasan et al. (2012) & 7 patients & Beta $(14-30 \mathrm{~Hz})$ \\
& 7 patients & Alpha $(7-10 \mathrm{~Hz})$ \\
& & \\
& & \\
Fraix et al. (2013) & Beta $(17.3-28.5 \mathrm{~Hz})$ \\
& & Alpha $(5-12 \mathrm{~Hz})$ \\
Tattersall et al. (2014) & Beta $(13-35 \mathrm{~Hz})$ \\
& & Gamma $(>35 \mathrm{~Hz})$ \\
Lau et al. (2015) & Alpha $(6-12 \mathrm{~Hz})$ \\
& Beta $(12-30 \mathrm{~Hz})$ \\
Valencia et al. (2014) & Alpha $(8-13 \mathrm{~Hz})$ \\
& 6 patients & Beta $(13-30 \mathrm{~Hz})$ \\
& Rats & Alpha $(8-12 \mathrm{~Hz})$ \\
& & Gamma $(30-70 \mathrm{~Hz})$
\end{tabular}

Findings or speculations

Play a physiological role

Play a physiological role

Related to attentional processes

Help to guide electrode implantation

Involved in sensory feedback

May be pro-kinetic

Correlate with gait performance

Suppress task irrelevant distraction

Mainly in the caudal-PPN

Mainly in the rostral-PPN

Pro-kinetic

Anti-kinetic

Anti-kinetic

Mainly in the caudal-PPN

Mainly in the rostral-PPN

Spatially localized within PPN

Spatially localized within PPN

Altered connectivity between motor cortex and the PPN 

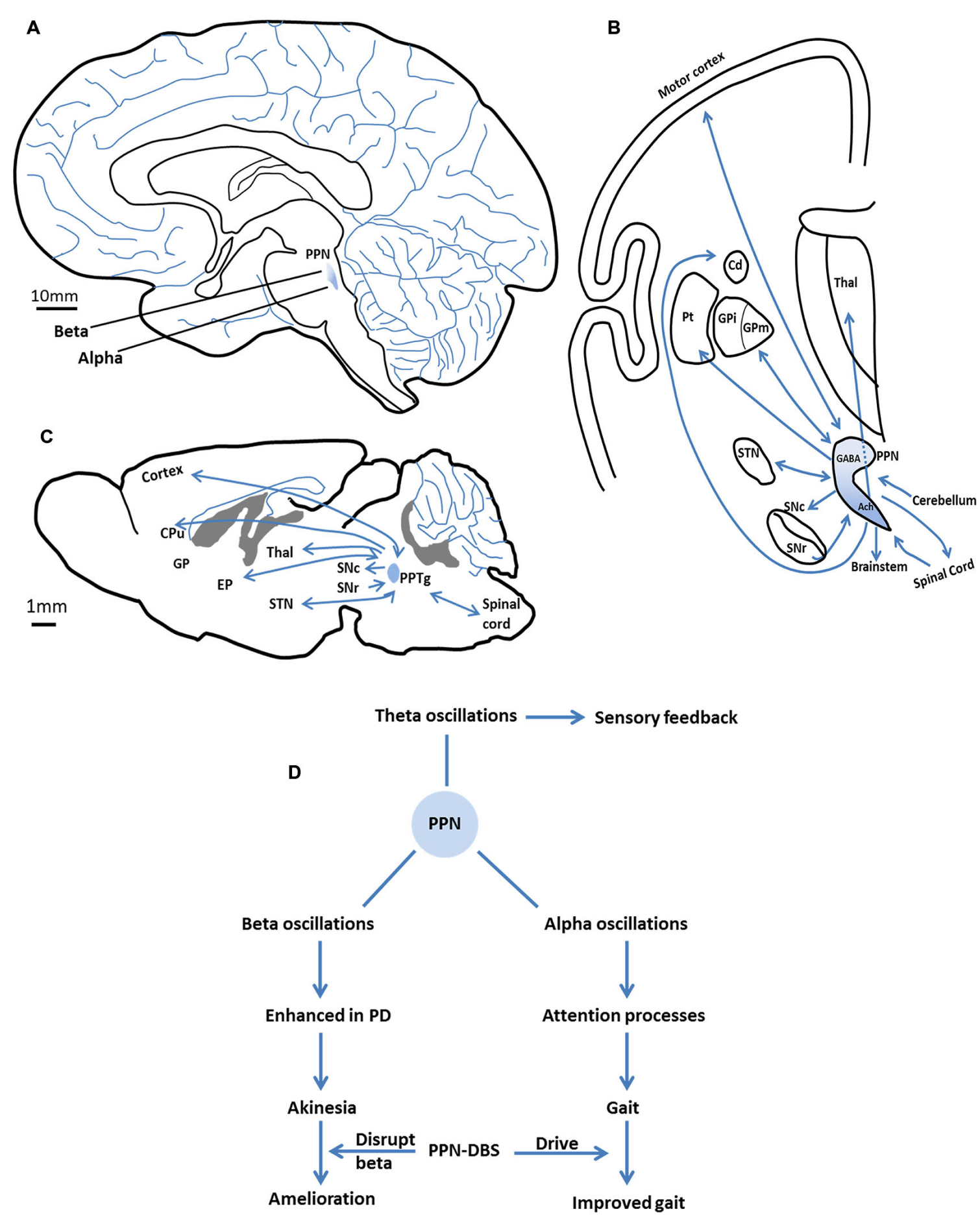

FIGURE 1 | The anatomy (location and connections) of the pedunculopontine nucleus (PPN) and the major oscillations in the PPN and their functional characteristics. (A) The location of the PPN in the human brain. Alpha oscillations mainly present in the caudal subregion of the PPN, beta oscillations predominantly in the rostral subregion. (B) The major efferent and afferent pathways of the PPN to the basal ganglia and other motor structures in the human brain. Neurons in the rostral subregion mainly express GABA, while neurons in the caudal subregion are primarily cholinergic. Adapted from Jenkinson et al. (2009). (C) The major efferent and afferent pathways of the
PPTg to the basal ganglia and other motor structures in the rat brain. (D) The major oscillations in the PPN and their functional characteristics. Alpha band promote gait performance by modulating attention processes. Enhanced beta band may result in akinesia. PPN-DBS may affect these two bands to improve symptoms. Theta band is involved in sensory feedback. SNc, substantia nigra compacta; SNr, substantia nigra reticular; GPi/EP, internal globus pallidus; GPm, medial pallidum; STN, subthalamic nucleus; $\mathrm{Cd} / \mathrm{CPu}$, caudate nucleus and putamen; Thal, thalamus; Pt, paratenial nucleus; GABA, gamma aminobutyric acid; Ach, acetylcholine. 


\section{Implications of PPN Oscillations for PD}

Alpha band power has been correlated with gait performance in parkinsonian patients, and oscillations at this frequency are increased after application of levodopa (Thevathasan et al., 2012; Fraix et al., 2013). Given this, one may wonder how alpha activity in the PPN might relate to the amelioration of axial symptoms. Alpha activity is considered to play a vital part in attention and in the allocation of processing resources in the brain (Klimesch, 1999; Palva and Palva, 2007). Increases in alpha activity may indicate the suppression of a distracting or task-irrelevant process, concomitant with the increased ability to focus attention on an important task (Ward, 2003; Jensen and Mazaheri, 2010). For example, alpha power increases with memory load during memory tasks, reflecting the individual's efforts to suppress distraction (Jensen et al., 2002). In the motor system, alpha activity is associated with the suppression of irrelevant processes in order to allow the smooth execution of motor programs (Pfurtscheller and Neuper, 1994; Suffczynski et al., 2001). Impairments in attentional function are common in parkinsonian patients (Wu and Hallett, 2008). Moreover, patients with axial symptoms (for example, gait freezing) are found to suffer more serious attentional impairments than those without Amboni et al. (2008) and Yogev-Seligmann et al. (2008). Thus, gait freezing is thought to be related to attentional deficits (Giladi and Hausdorff, 2006; Okuma, 2006). The results of a recent study on PPN-DBS of PD patients suggested that attentional augmentation can be "one possible mechanism to improve motor action and gait in patients with parkinsonian disorders" (Fischer et al., 2015). As part of the reticular activating system, the PPN could modulate attention via alpha oscillations to facilitate gait performance (Figure 1D).

Alpha oscillations have been recorded in a relatively caudal region of the PPN (Thevathasan et al., 2012), a region shown by animal studies to be rich in cholinergic neurons (Figures 1A,B; Martinez-Gonzalez et al., 2011). These neurons project widely, ranging from the cortex to locomotor centers (Figures 1B,C; Skinner et al., 1990; Mena-Segovia et al., 2008). Loss of cholinergic neurons in the PPN has been observed in PD, and this is thought to be associated with the gait and postural disorders of parkinsonian patients (Karachi et al., 2010; Grabli et al., 2013). Patients with a less severe loss of PPN cholinergic neurons may have better clinical outcomes with PPN-DBS therapy (Lau et al., 2015). Presumably, the dysfunction of cholinergic neurons might reduce the positive effects of PPN alpha activity in the modulation of attention processes, thereby leading to gait problems.

Theta rhythms in the PPN are hypothesized to be involved in the functional impact of sensory feedback, and not with other functions ascribed to the alpha band (Tsang et al., 2010). Based on animal studies, the PPN may be particularly involved in the sorting of sensory information for movement planning and performance (Winn, 2006). Studies of the phase relationship in theta band coherence between the cortex and PPN reveal that the sensorimotor cortex is temporally ahead of the PPN in motor planning, but is later in motor performing, suggesting that the cortex may drive the PPN in the phase of planning, then sensory information fed back to the cortex via the PPN to facilitate motor performance (Tsang et al., 2010). Coherence in theta activity has been observed in other related encephalic regions, such as the ipsilateral sensorimotor cortex and the ventral thalamus, areas that are suggested to form a system connecting related brain areas for the purpose of continuously monitoring sensory information (Nicolelis et al., 1995; Marsden et al., 2000). Thus, theta activity in the PPN may indicate that this region is part of this sensory feedback system (Figure 1D).

Within the PPN, neurons in the rostral subregion mainly express gamma aminobutyric acid (GABA) and are inhibitory, whereas neurons in the caudal subregion are primarily glutamatergic and cholinergic (Martinez-Gonzalez et al., 2011). These two regions have distinct projection patterns that connect them to disparate brain structures. Robust interconnections exist between neurons in the rostral PPN and the basal ganglia (MenaSegovia et al., 2004; Martinez-Gonzalez et al., 2011), whereas caudal PPN neurons mainly connect with cortical and locomotor centers such as the gait generator in the spinal cord (Figure 1B; Skinner et al., 1990; Mena-Segovia et al., 2008). This neuronal heterogeneity in the PPN likely underlies the differences in oscillatory activity between PPN subregions. For example, the beta activity recorded in the rostral subregion of the PPN is consistent with reciprocal connections to the basal ganglia, which exhibit excessive beta activity in PD. In the caudal PPN, alpha activity appears to be in coherence with cortical activity and relevant to locomotion.

The oscillatory topography discussed above suggests that the depth of recording electrodes along the rostro-caudal axis will have a significant effect on the type of dominant oscillatory patterns recorded in the PPN. This is one explanation for the discrepancy between studies that have recorded different oscillations in parkinsonian patients, which could lead to inconsistent interpretations of beta activity. The differing views regarding the importance of beta band oscillations in the PPN, however, likely also originate from additional factors such as small sample size, patient heterogeneity, and the diversity of analysis methods. According to animal data, increased beta oscillatory activity in the basal ganglia can be transmitted downstream to the PPN (Aravamuthan et al., 2008). Accordingly, from the current literature, it seems likely that beta activity in the human PPN, as in the basal ganglia, could contribute to akinesia in PD (Figure 1D), but more studies are needed.

\section{PPN Oscillations and PPN-DBS}

Although DBS has been used for decades for the treatment of PD and other movement disorders, the precise mechanisms underlying the efficacy of this therapy remain unclear. One speculation is that high frequency DBS of the STN inhibits neurons by focal depolarization, thereby blockading abnormal discharges from the basal ganglia (Filali et al., 2004; Welter et al., 2004; Chang et al., 2008). While this may be consistent with the classical Albin/Delong model (A widely accepted model of basal ganglia function based on the dominant anatomical 
connections of basal ganglia nuclei and their neurochemistry; Albin et al., 1989; DeLong, 1990), some doubt remains. For example, the electrical pulses used in DBS are thought to be too brief to blockade basal ganglia activity. Additionally, the long term suppression of basal ganglia activity should lead to the death of neurons and increased gliosis, yet these phenomena have not been observed in autopsies of DBS patients (Burbaud et al., 2002). A blockading effect is unlikely to be the mechanism underlying the efficacy of DBS in the PPN, since the low frequency stimulation used in this paradigm would not produce inhibitory effects.

Rather, it is possible that DBS of the PPN directly drives its activity to benefit parkinsonian states. The low frequency stimulus is the most effective for symptom relief in PPNDBS treatment (Mazzone et al., 2005), and this paradigm is generally considered to increase neuronal activity (Aravamuthan et al., 2008). The power of alpha oscillations recorded in the PPN of parkinsonian patients indicates that this activity may have a physiological function, since it is pathologically attenuated in PD. Therefore, low frequency stimulation of the PPN may drive the neurons and simulate the inherent alpha activity. This concept is supported by the interesting finding that parkinsonian patients who underwent PPN-DBS obtained the best therapeutic effect when the stimulation electrode was placed in the site of maximal alpha activity, namely, the caudal PPN (Thevathasan et al., 2012). Furthermore, nonhuman primate research suggests that the PPN is over-inhibited in parkinsonian states (Matsumura and Kojima, 2001; Nandi et al., 2002). Accordingly, the ability of DBS to drive the PPN is a likely mechanism for its therapeutic effects in PD (Figure 1D).

From recent studies, based upon recordings of pathological oscillatory activity (beta oscillations) in the cortical-basal ganglia loop in parkinsonian patients, it has been suggested that DBS may exert beneficial effects via the disruption of this aberrant activity. In the PPN, it is possible that DBS may exert its positive effects in this manner (Figure 1D). It has been suggested that pathological beta oscillations recorded in the PPN could have been transmitted downstream from the basal ganglia. Moreover, low frequency PPN-DBS was shown to reduce beta frequency activity in the STN of 6-OHDA lesioned rats (Alam et al., 2012).

Another mechanism, one that involves spike timing, could underlie the therapeutic effects of PPN-DBS. Studies of the relationship between PPN spiking and LFP activity found that the PPN spike timing changed significantly following 6-OHDA lesioning in rats. In intact rats, PPN firing tended to occur at the troughs of LFP oscillations, and this neuronal firing became peak-locked in the lesioned rats (Aravamuthan et al., 2008).

\section{References}

Alam, M., Heissler, H. E., Schwabe, K., and Krauss, J. K. (2012). Deep brain stimulation of the pedunculopontine tegmental nucleus modulates neuronal hyperactivity and enhanced beta oscillatory activity of the subthalamic nucleus in the rat 6-hydroxydopamine model. Exp. Neurol. 233, 233-242. doi: 10.1016/j.expneurol.2011. 10.006
Based on this evidence, it is possible that PPN-DBS affects PPN spike-timing relationship to normalize activity.

As mentioned, alpha and beta oscillations in the PPN are spatially localized (Thevathasan et al., 2012; Lau et al., 2015); thus this topographical arrangement can provide a guide for DBS electrode implantation. The finding that stimulation at the position of maximal alpha activity results in the most effective amelioration of symptoms suggests that the site of stimulation in the PPN is a crucial factor affecting therapeutic outcomes, one that merits more attention. Along these lines, a recent study on parkinsonian rats found distinct effects of the pedunculopontine tegmental nucleus (PPTg, the equivalent to the human PPN) DBS at different sites of stimulation, and emphasized "the critical importance of intra-PPTg DBS location" (Gut and Winn, 2015). The PPN is a heterogeneous structure in the brainstem that presents with an uncertainty boundary, and which is involved in multiple functions including cortical arousal, the control of behavioral processing, and locomotion (Ros et al., 2010; Benarroch, 2013). Different subregions in the PPN may have different functions (Martinez-Gonzalez et al., 2011). With regard to motor function, some processes may be closely associated with the cortico-basal ganglia loop, while others have connections with locomotion centers such as the gait generator in the spinal cord. The function of each subregion as well as their interconnections merits further study.

\section{Conclusion}

Studies that record oscillatory activity in the PPN have provided a great deal of information regarding the pathophysiology of $\mathrm{PD}$ and the mechanisms underlying the effects of PPN-DBS. Alpha activity is considered to be important in the PPN, where it may modulate attentional processes to improve gait performance in parkinsonian patients. DBS of the PPN could alleviate gait symptoms by driving PPN neurons and thereby affecting alpha oscillations. Nevertheless, PPN-DBS therapy remains experimental, and only a small number of patients are available to study. Large multicenter clinical trials are needed to best study the efficacy of PPN-DBS in PD. At the same time, more animal research would greatly supplement human studies by more mechanistic examination of this enigmatic brain region.

\section{Acknowledgments}

This work was supported by the National Natural Science Foundation of China (No. 81272150). The authors are very grateful to the assistance from The Guangdong Provincial Clinical Medical Center for Neurosurgery, No. 2013B020400005.

Albin, R. L., Young, A. B., and Penney, J. B. (1989). The functional anatomy of basal ganglia disorders. Trends Neurosci. 12, 366-375. doi: 10.1016/01662236(89)90074-x

Amboni, M., Cozzolino, A., Longo, K., Picillo, M., and Barone, P. (2008). Freezing of gait and executive functions in patients with Parkinson's disease. Mov. Disord. 23, 395-400. doi: 10.1002/mds.21850

Androulidakis, A. G., Khan, S., Litvak, V., Pleydell-Pearce, C. W., Brown, P., and Gill, S. S. (2008a). Local field potential recordings from the pedunculopontine 
nucleus in a Parkinsonian patient. Neuroreport 19, 59-62. doi: 10.1097/wnr. 0b013e3282f2e2d1

Androulidakis, A. G., Mazzone, P., Litvak, V., Penny, W., Dileone, M., Gaynor, L. M., et al. (2008b). Oscillatory activity in the pedunculopontine area of patients with Parkinson's disease. Exp. Neurol. 211, 59-66. doi: 10.1016/j. expneurol.2008.01.002

Aravamuthan, B. R., Bergstrom, D. A., French, R. A., Taylor, J. J., Parr-Brownlie, L. C., and Walters, J. R. (2008). Altered neuronal activity relationships between the pedunculopontine nucleus and motor cortex in a rodent model of Parkinson's disease. Exp. Neurol. 213, 268-280. doi: 10.1016/j.expneurol.2008. 05.023

Benarroch, E. E. (2013). Pedunculopontine nucleus: functional organization and clinical implications. Neurology 80, 1148-1155. doi: 10.1212/wnl. 0b013e3182886a76

Boraud, T., Brown, P., Goldberg, J., Graybiel, A., and Magill, P. (2005). "Oscillations in the basal ganglia: the good, the bad and the unexpected," in The Basal Ganglia VIII, eds J. P. Bolam, C. Ingham, and P. Magill (New York: Springer Science and Business Media), 1-24.

Brown, P. (2003). Oscillatory nature of human basal ganglia activity: relationship to the pathophysiology of Parkinson's disease. Mov. Disord. 18, 357-363. doi: $10.1002 / \mathrm{mds} .10358$

Brown, P., Oliviero, A., Mazzone, P., Insola, A., Tonali, P., and Di Lazzaro, V. (2001). Dopamine dependency of oscillations between subthalamic nucleus and pallidum in Parkinson's disease. J. Neurosci. 21, 1033-1038.

Burbaud, P., Vital, A., Rougier, A., Bouillot, S., Guehl, D., Cuny, E., et al. (2002). Minimal tissue damage after stimulation of the motor thalamus in a case of chorea-acanthocytosis. Neurology 59, 1982-1984. doi: 10.1212/01.wnl. 0000038389.30437.1e

Chang, J. Y., Shi, L. H., Luo, F., Zhang, W. M., and Woodward, D. J. (2008). Studies of the neural mechanisms of deep brain stimulation in rodent models of Parkinson's disease. Neurosci. Biobehav. Rev. 32, 352-366. doi: 10.1016/j. neubiorev.2007.09.002

Chen, C. C., Litvak, V., Gilbertson, T., Kühn, A., Lu, C. S., Lee, S. T., et al. (2007). Excessive synchronization of basal ganglia neurons at $20 \mathrm{~Hz}$ slows movement in Parkinson's disease. Exp. Neurol. 205, 214-221. doi: 10.1016/j.expneurol.2007. 04.003

DeLong, M. R. (1990). Primate models of movement disorders of basal ganglia origin. Trends Neurosci. 13, 281-285. doi: 10.1016/0166-2236(90) 90110-v

Eusebio, A., and Brown, P. (2007). Oscillatory activity in the basal ganglia. Parkinsonism Relat. Disord. 13(Suppl. 3), S434-S436. doi: 10.1016/S13538020(08)70044-0

Filali, M., Hutchison, W. D., Palter, V. N., Lozano, A. M., and Dostrovsky, J. O. (2004). Stimulation-induced inhibition of neuronal firing in human subthalamic nucleus. Exp. Brain Res. 156, 274-281. doi: 10.1007/s00221-003$1784-y$

Fischer, J., Schwiecker, K., Bittner, V., Heinze, H. J., Voges, J., Galazky, I., et al. (2015). Modulation of attentional processing by deep brain stimulation of the pedunculopontine nucleus region in patients with Parkinsonian disorders. Neuropsychology 29, 632-637. doi: 10.1037/neu0000179

Fogelson, N., Williams, D., Tijssen, M., van Bruggen, G., Speelman, H., and Brown, P. (2006). Different functional loops between cerebral cortex and the subthalmic area in Parkinson's disease. Cereb. Cortex 16, 64-75. doi: 10. 1093/cercor/bhi084

Fraix, V., Bastin, J., David, O., Goetz, L., Ferraye, M., Benabid, A. L., et al. (2013). Pedunculopontine nucleus area oscillations during stance, stepping and freezing in Parkinson's disease. PLoS One 8:e83919. doi: 10.1371/journal.pone. 0083919

Gatev, P., Darbin, O., and Wichmann, T. (2006). Oscillations in the basal ganglia under normal conditions and in movement disorders. Mov. Disord. 21, 1566-1577. doi: $10.1002 / \mathrm{mds} .21033$

Giladi, N., and Hausdorff, J. M. (2006). The role of mental function in the pathogenesis of freezing of gait in Parkinson's disease. J. Neurol. Sci. 248, 173-176. doi: 10.1016/j.jns.2006.05.015

Grabli, D., Karachi, C., Folgoas, E., Monfort, M., Tande, D., Clark, S., et al. (2013). Gait disorders in parkinsonian monkeys with pedunculopontine nucleus lesions: a tale of two systems. J. Neurosci. 33, 11986-11993. doi: 10. 1523/jneurosci.1568-13.2013
Gut, N. K., and Winn, P. (2015). Deep brain stimulation of different pedunculopontine targets in a novel rodent model of parkinsonism. J. Neurosci. 35, 4792-4803. doi: 10.1523/JNEUROSCI.3646-14.2015

Hammond, C., Bergman, H., and Brown, P. (2007). Pathological synchronization in Parkinson's disease: networks, models and treatments. Trends Neurosci. 30, 357-364. doi: 10.1016/j.tins.2007.05.004

Jenkinson, N., Nandi, D., Muthusamy, K., Ray, N. J., Gregory, R., Stein, J. F., et al. (2009). Anatomy, physiology and pathophysiology of the pedunculopontine nucleus. Mov. Disord. 24, 319-328. doi: 10.1002/mds.22189

Jensen, O., Gelfand, J., Kounios, J., and Lisman, J. E. (2002). Oscillations in the alpha band $(9-12 \mathrm{~Hz})$ increase with memory load during retention in a shortterm memory task. Cereb. Cortex 12, 877-882. doi: 10.1093/cercor/12.8.877

Jensen, O., and Mazaheri, A. (2010). Shaping functional architecture by oscillatory alpha activity: gating by inhibition. Front. Hum. Neurosci. 4:186. doi: 10 . 3389/fnhum.2010.00186

Karachi, C., Grabli, D., Bernard, F. A., Tande, D., Wattiez, N., Belaid, H., et al. (2010). Cholinergic mesencephalic neurons are involved in gait and postural disorders in Parkinson disease. J. Clin. Invest. 120, 2745-2754. doi: 10. $1172 /$ JCI 42642

Klimesch, W. (1999). EEG alpha and theta oscillations reflect cognitive and memory performance: a review and analysis. Brain Res. Brain Res. Rev. 29, 169-195. doi: 10.1016/s0165-0173(98)00056-3

Lau, B., Welter, M. L., Belaid, H., Fernandez Vidal, S., Bardinet, E., Grabli, D., et al. (2015). The integrative role of the pedunculopontine nucleus in human gait. Brain 138, 1284-1296. doi: 10.1093/brain/awv047

Levy, R., Ashby, P., Hutchison, W. D., Lang, A. E., Lozano, A. M., and Dostrovsky, J. O. (2002). Dependence of subthalamic nucleus oscillations on movement and dopamine in Parkinson's disease. Brain 125, 1196-1209. doi: 10. 1093/brain/awf128

Marsden, J. F., Ashby, P., Limousin-Dowsey, P., Rothwell, J. C., and Brown, P. (2000). Coherence between cerebellar thalamus, cortex and muscle in man: cerebellar thalamus interactions. Brain 123, 1459-1470. doi: 10.1093/brain/123. 7.1459

Martinez-Gonzalez, C., Bolam, J. P., and Mena-Segovia, J. (2011). Topographical organization of the pedunculopontine nucleus. Front. Neuroanat. 5:22. doi: 10 . 3389/fnana.2011.00022

Matsumura, M., and Kojima, J. (2001). The role of the pedunculopontine tegmental nucleus in experimental parkinsonism in primates. Stereotact. Funct. Neurosurg. 77, 108-115. doi: 10.1159/000064614

Mazzone, P., Lozano, A., Stanzione, P., Galati, S., Scarnati, E., Peppe, A., et al. (2005). Implantation of human pedunculopontine nucleus: a safe and clinically relevant target in Parkinson's disease. Neuroreport 16, 1877-1881. doi: 10. 1097/01.wnr.0000187629.38010.12

Mena-Segovia, J., Bolam, J. P., and Magill, P. J. (2004). Pedunculopontine nucleus and basal ganglia: distant relatives or part of the same family? Trends Neurosci. 27, 585-588. doi: 10.1016/j.tins.2004.07.009

Mena-Segovia, J., Sims, H. M., Magill, P. J., and Bolam, J. P. (2008). Cholinergic brainstem neurons modulate cortical gamma activity during slow oscillations. J. Physiol. 586, 2947-2960. doi: 10.1113/jphysiol.2008.153874

Morita, H., Hass, C. J., Moro, E., Sudhyadhom, A., Kumar, R., and Okun, M. S. (2014). Pedunculopontine nucleus stimulation: where are we now and what needs to be done to move the field forward? Front. Neurol. 5:243. doi: 10 . 3389/fneur.2014.00243

Moro, E., Hamani, C., Poon, Y. Y., Al-Khairallah, T., Dostrovsky, J. O., Hutchison, W. D., et al. (2010). Unilateral pedunculopontine stimulation improves falls in Parkinson's disease. Brain 133, 215-224. doi: 10.1093/brain/awp261

Nandi, D., Aziz, T. Z., Giladi, N., Winter, J., and Stein, J. F. (2002). Reversal of akinesia in experimental parkinsonism by GABA antagonist microinjections in the pedunculopontine nucleus. Brain 125, 2418-2430. doi: 10.1093/brain/awf259

Nicolelis, M. A., Baccala, L. A., Lin, R. C., and Chapin, J. K. (1995). Sensorimotor encoding by synchronous neural ensemble activity at multiple levels of the somatosensory system. Science 268, 1353-1358. doi: 10.1126/science. 7761855

Okuma, Y. (2006). Freezing of gait in Parkinson's disease. J. Neurol. 253, VII27-VII32. doi: 10.1016/j.gaitpost.2014.09.021

Palva, S., and Palva, J. M. (2007). New vistas for alpha-frequency band oscillations. Trends Neurosci. 30, 150-158. doi: 10.1016/j.tins.2007.02.001 
Pfurtscheller, G., and Neuper, C. (1994). Event-related synchronization of mu rhythm in the EEG over the cortical hand area in man. Neurosci. Lett. 174, 93-96. doi: 10.1016/0304-3940(94)90127-9

Ros, H., Magill, P. J., Moss, J., Bolam, J. P., and Mena-Segovia, J. (2010). Distinct types of non-cholinergic pedunculopontine neurons are differentially modulated during global brain states. Neuroscience 170, 78-91. doi: 10.1016/j. neuroscience.2010.06.068

Shimamoto, S. A., Larson, P. S., Ostrem, J. L., Glass, G. A., Turner, R. S., and Starr, P. A. (2010). Physiological identification of the human pedunculopontine nucleus. J. Neurol. Neurosurg. Psychiatry 81, 80-86. doi: 10.1136/jnnp.2009. 179069

Skinner, R. D., Kinjo, N., Henderson, V., and Garcia-Rill, E. (1990). Locomotor projections from the pedunculopontine nucleus to the spinal cord. Neuroreport 1, 183-186. doi: 10.1097/00001756-199011000-00001

Stein, E., and Bar-Gad, I. (2013). beta oscillations in the cortico-basal ganglia loop during parkinsonism. Exp. Neurol. 245, 52-59. doi: 10.1016/j.expneurol.2012. 07.023

Suffczynski, P., Kalitzin, S., Pfurtscheller, G., and Lopes da Silva, F. H. (2001). Computational model of thalamo-cortical networks: dynamical control of alpha rhythms in relation to focal attention. Int. J. Psychophysiol. 43, 25-40. doi: 10.1016/s0167-8760(01)00177-5

Tattersall, T. L., Stratton, P. G., Coyne, T. J., Cook, R., Silberstein, P., Silburn, P. A., et al. (2014). Imagined gait modulates neuronal network dynamics in the human pedunculopontine nucleus. Nat. Neurosci. 17, 449-454. doi: 10. 1038/nn. 3642

Thevathasan, W., Coyne, T. J., Hyam, J. A., Kerr, G., Jenkinson, N., Aziz, T. Z., et al. (2011). Pedunculopontine nucleus stimulation improves gait freezing in Parkinson disease. Neurosurgery 69, 1248-1253; discussion 1254. doi: 10. 1227/neu.0b013e31822b6f71

Thevathasan, W., Pogosyan, A., Hyam, J. A., Jenkinson, N., Foltynie, T., Limousin, P., et al. (2012). Alpha oscillations in the pedunculopontine nucleus correlate with gait performance in parkinsonism. Brain 135, 148-160. doi: 10 . 1093/brain/awr315

Tsang, E. W., Hamani, C., Moro, E., Mazzella, F., Poon, Y. Y., Lozano, A. M., et al. (2010). Involvement of the human pedunculopontine nucleus region in voluntary movements. Neurology 75, 950-959. doi: 10.1212/WNL. 0b013e3181f25b35

Uhlhaas, P. J., and Singer, W. (2006). Neural synchrony in brain disorders: relevance for cognitive dysfunctions and pathophysiology. Neuron 52, 155-168. doi: 10.1016/j.neuron.2006.09.020

Valencia, M., Chavez, M., Artieda, J., Bolam, J. P., and Mena-Segovia, J. (2014). Abnormal functional connectivity between motor cortex and pedunculopontine nucleus following chronic dopamine depletion. J. Neurophysiol. 111, 434-440. doi: 10.1152/jn.00555.2013

Ward, L. M. (2003). Synchronous neural oscillations and cognitive processes. Trends Cogn. Sci. 7, 553-559. doi: 10.1016/j.tics.2003. 10.012

Weinberger, M., Hamani, C., Hutchison, W. D., Moro, E., Lozano, A. M., and Dostrovsky, J. O. (2008). Pedunculopontine nucleus microelectrode recordings in movement disorder patients. Exp. Brain Res. 188, 165-174. doi: 10. 1007/s00221-008-1349-1

Weinberger, M., Mahant, N., Hutchison, W. D., Lozano, A. M., Moro, E., Hodaie, M., et al. (2006). Beta oscillatory activity in the subthalamic nucleus and its relation to dopaminergic response in Parkinson's disease. J. Neurophysiol. 96, 3248-3256. doi: 10.1152/jn.00697.2006

Welter, M. L., Houeto, J. L., Bonnet, A. M., Bejjani, P. B., Mesnage, V., Dormont, D., et al. (2004). Effects of high-frequency stimulation on subthalamic neuronal activity in parkinsonian patients. Arch. Neurol. 61, 89-96. doi: 10. 1001/archneur.61.1.89

Wilcox, R. A., Cole, M. H., Wong, D., Coyne, T., Silburn, P., and Kerr, G. (2011). Pedunculopontine nucleus deep brain stimulation produces sustained improvement in primary progressive freezing of gait. J. Neurol. Neurosurg. Psychiatry 82, 1256-1259. doi: 10.1136/jnnp.2010. 213462

Winn, P. (2006). How best to consider the structure and function of the pedunculopontine tegmental nucleus: evidence from animal studies. J. Neurol. Sci. 248, 234-250. doi: 10.1016/j.jns.2006.05.036

Wu, T., and Hallett, M. (2008). Neural correlates of dual task performance in patients with Parkinson's disease. J. Neurol. Neurosurg. Psychiatry 79, 760-766. doi: 10.1136/jnnp.2007.126599

Yogev-Seligmann, G., Hausdorff, J. M., and Giladi, N. (2008). The role of executive function and attention in gait. Mov. Disord. 23, 329-342. doi: 10.1002/mds. 21720

Conflict of Interest Statement: The authors declare that the research was conducted in the absence of any commercial or financial relationships that could be construed as a potential conflict of interest.

Copyright (C) $2015 \mathrm{Li}$ and Zhang. This is an open-access article distributed under the terms of the Creative Commons Attribution License (CC BY). The use, distribution and reproduction in other forums is permitted, provided the original author(s) or licensor are credited and that the original publication in this journal is cited, in accordance with accepted academic practice. No use, distribution or reproduction is permitted which does not comply with these terms. 